\title{
Monitoramento de Fluxo de Oxigênio para Bombas Respiratórias de Ventiladores Pulmonares
}

\author{
Vitor Emanuel Batista $^{1}$, Janine Kniess ${ }^{1}$ \\ ${ }^{1}$ Programa de Pós-Graduação em Computação Aplicada - PPGCA \\ Universidade do Estado de Santa Catarina - UDESC - Joinville - SC - Brazil \\ vitor.ebatista@gmail.com, janine.kniess@udesc.br
}

\begin{abstract}
The Severe Acute Respiratory Syndrome Coronavirus 2 (SARS-CoV2) increased the number of patients with infections in the respiratory system. Thereby, the demand for mechanical ventilators has grown. Accurate monitoring of oxygen flow and volume is essential to minimize ventilator-induced lung injury (VILI). Systems for monitoring the oxygen flow and to generate alerts are vital to the qualification of the equipment. This paper presents a hardware and software architecture that works with a pulmonary ventilator. The system collects the oxygen flow rate sent to patients and transmits the captured information via MQTT protocol. Based on the observed data, the system also generates sound and visual alerts for the medical team.
\end{abstract}

Resumo. A Síndrome Respiratória Aguda Grave 2 (SARS-CoV-2) aumentou o número de pacientes com infecções respiratórias. Com isso, cresceu a demanda por ventiladores pulmonares. O monitoramento preciso do fluxo e das trocas de volume de oxigênio é essencial para minimizar lesões pulmonares induzidas pelo ventilador. Sistemas para monitorar o fluxo de oxigênio e gerar alertas são vitais para a qualificação do equipamento. Neste artigo, apresenta-se uma arquitetura de hardware e software que atua junto a um ventilador pulmonar. $O$ sistema coleta o fluxo de vazão de oxigênio gerado para um paciente e envia via protocolo MQTT as informações capturadas. Com base nos dados observados, o sistema também gera alertas sonoros e visuais para a equipe médica.

\section{Introdução}

A Síndrome Respiratória Aguda Grave (SARS-CoV-2) é o agente causador do Coronavírus 2019 (COVID-19), que foi declarada uma pandemia pela Organização Mundial da Saúde (OMS) em março de 2020. A COVID-19 é altamente contagiosa e leva principalmente a uma infecção do sistema respiratório [Yang et al. 2020].

A ventilação mecânica é um sistema de suporte à vida usado para manter a função pulmonar adequada em pacientes gravemente enfermos ou sob anestesia geral [Silva and Rocco ]. No tratamento da COVID-19 os ventiladores mecânicos precisam fornecer com precisão a quantidade necessária de gases para evitar o aumento da incidência de lesão pulmonar induzida pelo ventilador. Os medidores de vazão embutidos nos ventiladores devem fornecer dados em tempo real para o ajuste do fluxo e volume de gases fornecido ao pacientes [Schena et al. 2015]. O monitoramento preciso da taxa de fluxo e das trocas de volume é essencial para minimizar as lesões pulmonares. Como os medidores de vazão desempenham um papel essencial nesse campo, eles devem cumprir 
critérios rigorosos em termos de características dinâmicas (resposta de frequência adequada e tempo de resposta curto) e estáticas (boa precisão e resolução, alta sensibilidade e razão de abertura adequada) [Schena et al. 2015].

Neste trabalho, propõe-se um dispositivo de baixo custo que pode ser integrado a um equipamento de processo respiratório, para auxiliar na análise dos dados de respiração. No desenvolvimento do dispositivo, adotou-se a especificação proposta pelo governo do Reino Unido de um sistema de ventilação rapidamente manufaturado, do inglês, Rapidly Manufactured Ventilator System (RMVS) [UK-Government 2020]. O dispositivo gera alertas quando identifica um padrão não aceitável, visando auxiliar no monitoramento remoto e minimizar o contato físico.

Este artigo está organizado nas seguintes seções: na Seção 2 são apresentados os trabalhos relacionados, na Seção 3, a abordagem proposta e sua arquitetura. Na Seção 4 os resultados obtidos com o experimentos e por fim, na Seção 5 as conclusões do trabalho.

\section{Trabalhos Relacionados}

Segundo a Agência Nacional de Vigilância Sanitária [Anvisa 2020], a Ventilação Mecânica (VM) ocorre através de aparelhos que, intermitentemente, insuflam as vias respiratórias com volumes de ar. O movimento do gás oxigênio para dentro dos pulmões ocorre devido à geração de um gradiente de pressão entre as vias aéreas superiores e o alvéolo, podendo ser conseguido por um equipamento que diminua a pressão alveolar ou que aumente a pressão da via aérea proximal. O deslocamento do ar ambiente para o interior dos pulmões e de lá para o exterior acontece por meio de ventiladores pulmonares. A Anvisa ainda relata que, a depender de como o ventilador é indicado e regulado, ele influi de forma favorável ou não na ventilação e mecanismos de troca gasosa do paciente, contribuindo para a manutenção da vida ou para a degradação da sua condição clínica. Com base nas recomendações apresentadas pela Anvisa e seguindo os requisitos do governo do Reino Unido [UK-Government 2020], neste trabalho, apresenta-se uma abordagem para suporte aos ventiladores pulmonares, com o objetivo de realizar o monitoramento do fluxo de ar dos ventiladores pulmonares e reduzir consequentes lesões.

$\mathrm{Na}$ literatura, existem vários trabalhos que visam calibrar os ventiladores pulmonares e assim reduzir a incidência de lesões nos pacientes. Em Uechi (2012), apresenta-se uma metodologia para boas práticas de calibração destes equipamentos, bem como um conjunto de experimentos de calibração com ventiladores pulmonares reais [Uechi et al. 2012]. Os resultados comprovaram que essa prática melhorou a qualidade da assistência. No trabalho de Silva (2007), os autores propõem protótipo para ensaio de desempenho de ventiladores pulmonares [Silva and Moraes 2007]. O sistema obtém formas de onda de pressão nas vias aéreas e fluxo de ar, fornecidos pelo ventilador, através de um microcontrolador e envia os dados amostrados para um PC via Bluetooth. Os sensores de pressão e fluxo foram calibrados utilizando como padrão de referência equipamentos com calibração rastreável. Testes com ventiladores pulmonares foram feitos para validar o sistema. Diferente de Silva (2007), o sistema apresentado neste trabalho, provê uma arquitetura que permite a análise offline dos dados e o monitoramento remoto através de um aparelho celular. Acrescenta-se o custo reduzido do sistema e uso de protocolos de IoT como o MQTT, que busca a economia de bateria dos dispositivos com um mecanismo eficiente de troca de mensagens. 


\section{Abordagem Proposta}

Como a medição e monitoramento de vazão em ventiladores são essenciais para o suporte a vida, o governo do Reino Unido, com apoio de profissionais de anestesia, medicina intensiva e reguladores de dispositivos médicos, apresentou uma especificação de ventiladores para uso em hospitais durante o surto de coronavirus [UK-Government 2020]. Esta especificação é a mais importante e utilizada por pesquisadores, sendo assim, os seguintes requisitos são baseados no sistema:

1. Prover pelo menos duas configurações para o volume de ar (p.ex. $450 \mathrm{ml}$ ou 350 $\mathrm{ml}$ ). Permitir uma taxa ajustável entre 10 e 30 ciclos/respirações por minuto.

2. Permitir a configuração do volume corrente, se fornecido. Com relação ao volume de gás que flui para os pulmões durante um ciclo inspiratório deve-se: Oferecer ao menos uma configuração entre $10 \mathrm{ml}$ e $400 \mathrm{ml}$ de volume de ar; Considerar um intervalo de $250 \mathrm{ml}$ a $600 \mathrm{ml}$ de volume de ar em etapas de $50 \mathrm{ml}$ por ciclo; Permitir um alcance de até $800 \mathrm{ml}$ de volume de ar por ciclo.

3. Enviar pelo menos $400 \mathrm{ml}$ de mistura ar em até 1,5 segundos.

4. Funcionar continuamente sem falhas e alertar caso o volume corrente não for alcançado ou for excedido.

\subsection{Arquitetura da Aplicação}

A integração entre o hardware proposto com o ventilador pulmonar ocorre através de um sensor de fluxo de vazão integrado a uma mangueira de circulação de ar. O ar é gerado a partir de uma bomba (RMVS), e circula por um mangueira até o sensor de fluxo pela mangueira até o paciente. O sensor de fluxo mede o nível de ar gerado e o envia para um microcontrolador conectado a uma rede IEEE 802.11. Os dados obtidos pelo sensor de fluxo são enviados pela rede sem fio via o protocolo da Camada de Aplicação Message Queuing Telemetry Transport (MQTT). Os dados são analisados por um servidor remoto e retornam para o microcontrolador que gera alertas sonoros e visuais. Um aplicativo para celular foi desenvolvido com o objetivo de apresentar os dados do fluxo. A arquitetura do sistema é apresentada na Figura 1.

No desenvolvimento do protótipo apresentado na Seção 4, utilizou-se o hardware conforme esquematizado na Figura 2: um buzzer ativo para emissão de um sinal sonoro para a equipe médica, LEDs para visualização do estado do fluxo, um sensor de efeito Hall (YF-S201) para medição do fluxo e por fim um módulo ESP8266 NodeMCU (ESP-12E) que executa somente a aplicação.

A medição do fluxo de oxigênio funciona a partir do sensor de efeito Hall, que tem como base um sensor magnético preso a uma engrenagem. A engrenagem mede com precisão a rotação, ou seja, quando o oxigênio passar pela tubulação e entrar no sensor, rotacionará com a força do fluxo. Após esta etapa, envia por meio de pulsos informações ao microcontrolador para que o dispositivo realize o processamento e cálculo do volume de oxigênio gerado pelo respirador. A frequência dos pulsos gerados é proporcional a velocidade de rotação e esta por sua vez é compatível com o fluxo de ar que a impulsiona [Sharon et al. 2018]. A escolha deste sensor foi pelo baixo custo e fácil integração para análise dos dados, contudo, sua baixa precisão para um fim médico é um ponto de atenção.

As informações coletadas pelo sensor e processadas pelo microcontrolador, são enviadas para um servidor remoto via o protocolo MQTT. O servidor é encarregado de 


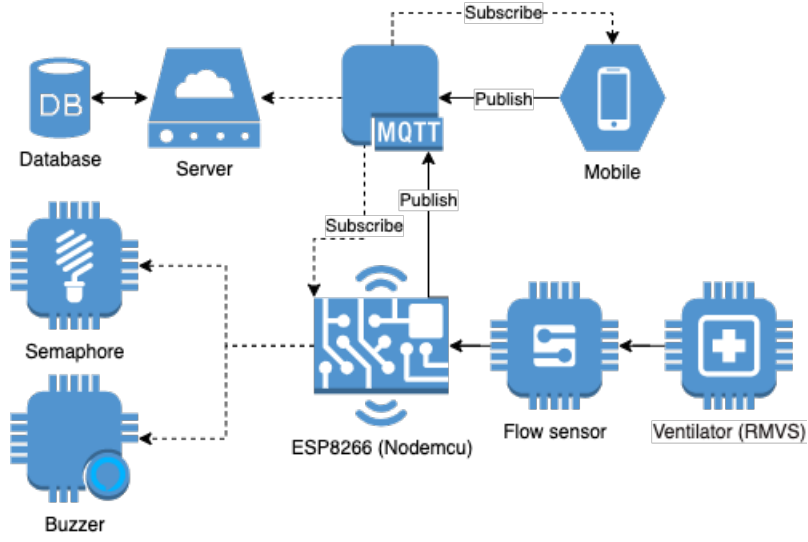

Figura 1. Arquitetura do Sistema.

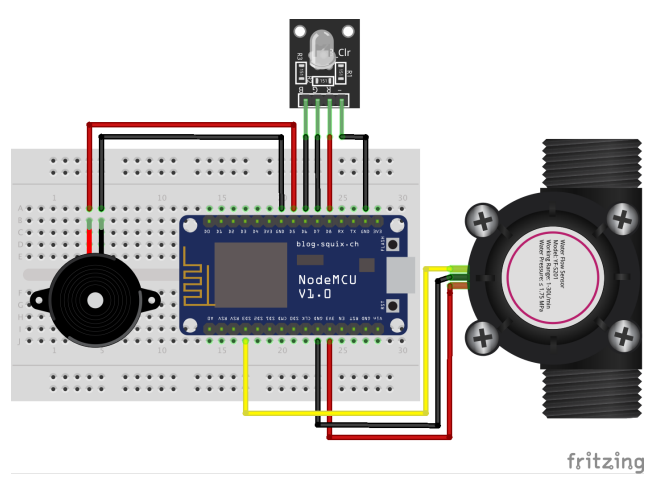

Figura 2. Esquema de Funcionamento da Aplicação.

processar as informações recebidas, armazenar no banco de dados e processar por meio de um algoritmo os requisitos descritos na Seção 3. Além disso, os dados históricos armazenados no banco de dados podem auxiliar na tomada de decisões.

O MQTT é um protocolo padrão aberto OASIS e ISO (ISO/IEC 20922) que adota o modelo de publicação e assinatura (publish-subscribe), sendo leve e projetado para equipamentos restritos, o que é ideal para dispositivos de IoT [Liu et al. 2020]. Além disso, o MQTT oferece o mais alto nível de qualidade de serviço e é mais adequado para grandes redes de pequenos dispositivos que precisam ser monitorados ou controlados a partir de um servidor [Naik 2017]. Na arquitetura proposta na Figura 1, o cliente MQTT no microcontrolador estabelece uma conexão com o servidor (Broker) MQTT, publica e subscreve os tópicos de acordo com a necessidade da aplicação.

\section{Resultados}

No desenvolvimento do dispositivo de hardware, utilizou-se um sensor de fluxo, semáforos de LED, um buzzer ativo de $5 \mathrm{~V}$ para emissão do sinal sonoro e um microcontrolador do tipo ESP8266 NodeMCU. Os dados capturados do sensor de fluxo são enviados via o protocolo MQTT para um servidor com a tecnologia Node.JS. Os dados são armazenados em banco de dados MongoDB.

Nos testes, foi utilizada uma bomba manual para simular o ventilador pulmonar. A bomba manual gera o oxigênio que circula em uma mangueira que está conectada ao sensor de fluxo, o que faz acionar e gerar dados. O circuito apresentado na Figura 3, foi organizado conforme o diagrama apresentado na Figura 1. Quando o sensor de fluxo está desconectado da mangueira por onde o oxigênio passa ou a bomba não gera oxigênio suficiente ao paciente, o semáforo aciona o LED vermelho. Neste caso, o sistema sinaliza que o valor de volume de oxigênio não corresponde aos requisitos mínimos apresentados na Seção 3.

O semáforo muda de cor, vermelho, amarelo e verde para representar o status do sistema a saber: irregular, atenção e normal, respectivamente. Além disso, um sinal sonoro é acionado sempre que o sistema muda para status vermelho. Para a equipe médica, o sinal sonoro e a mudança de cor no semáforo podem não ser suficientes. Desta forma, um aplicativo para celular que informa a equipe médica sobre as condições do paciente foi integrado ao sistema. Optou-se pelo aplicativo IoT OnOff [OnOff 2020]. Na Figura 4, 

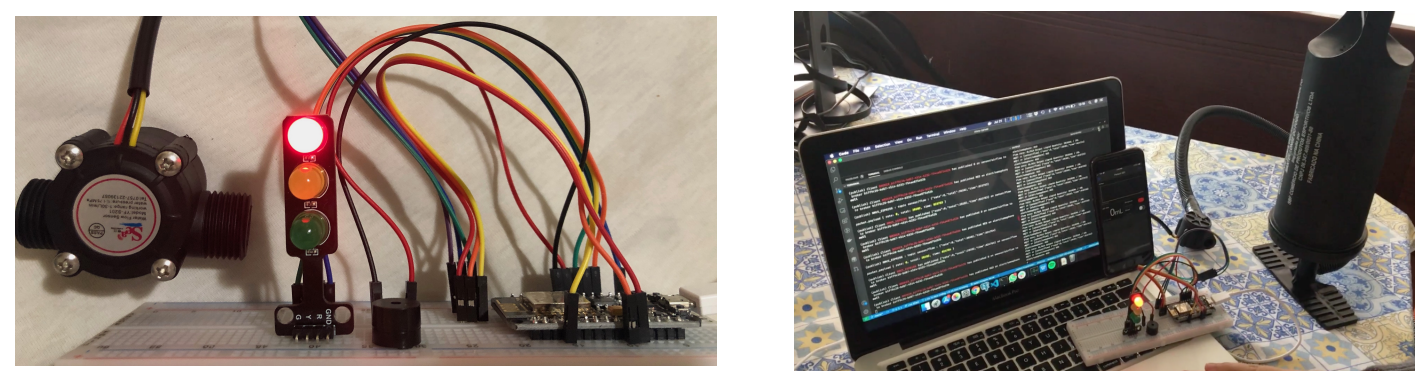

Figura 3. Circuito do Projeto

apresenta-se três cenários, um para cada status possível do sistema. Em todos os cenários o fluxo de oxigênio atual e o histórico é mostrado (subscribe). O usuário do sistema também pode acionar (publish) à distância o buzzer e alertar com um sinal sonoro algum profissional que esteja próximo ao paciente.
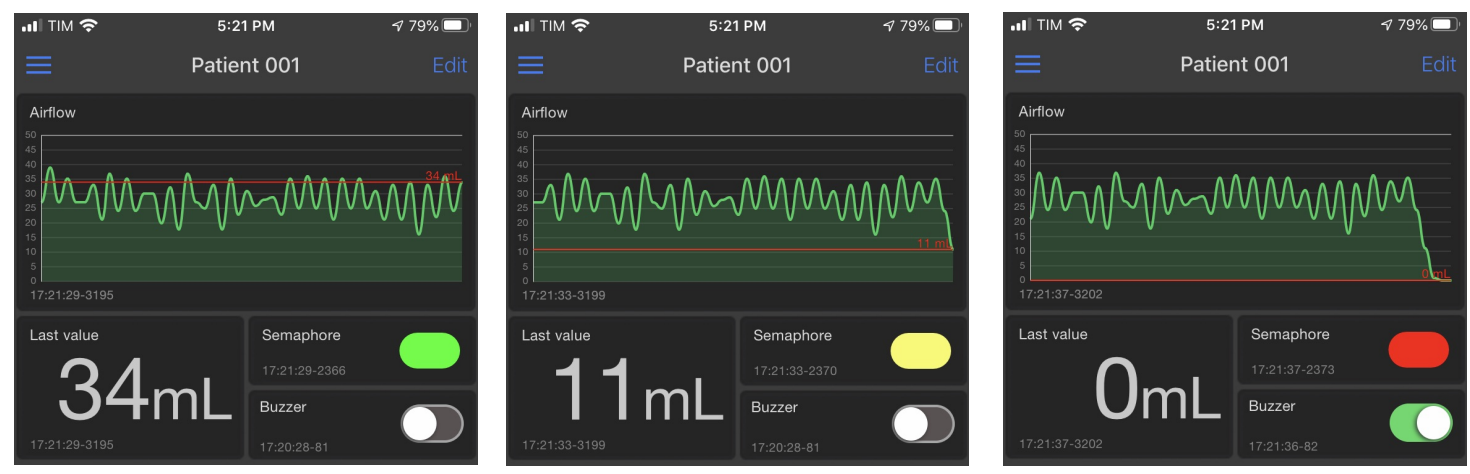

Figura 4. Monitoramento por celular.

A biblioteca Bree foi responsável por gerenciar as tarefas e controlar a execução autônoma de funções em segundo plano no servidor Node.js, onde os dados são avaliados e armazenados, além de gerar alertas conforme requisitos descritos na Seção 3. A tecnologia de comunicação usada nos testes é o IEEE 802.11. Por fim, a possibilidade de consultar os dados e receber alertas de forma instantânea via um aplicativo desenvolvido para celular é um diferencial para uma rápida atuação da equipe médica.

\section{Conclusão}

A ventilação mecânica pulmonar é fundamental para a sobrevivência de pacientes graves do COVID-19, bem como para as demais doenças em que pacientes possuem alguma insuficiência respiratória grave. Estes ventiladores mecânicos precisam fornecer com precisão a quantidade necessária de gases para evitar o aumento da incidência de lesão pulmonar. Os medidores de vazão embutidos nos ventiladores devem fornecer informações em tempo real para ajustar fluxo e volume de gases enviados aos pacientes.

Neste artigo foi desenvolvido um protótipo em hardware e software que permite o monitoramento do fluxo de vazão de oxigênio em ventiladores pulmonares. O sistema transmite os dados via protocolo MQTT, o que permite gerar alertas sonoros e visuais em tempo real quando algum dos requisitos essenciais do sistema for descumprido. $O$ MQTT mostrou na arquitetura do sistema, eficiente na captura, processamento e emissão 
de alertas em tempo real. Por fim, uma aplicação para celular foi desenvolvida para auxiliar a equipe médica quanto ao acompanhamento remoto do paciente.

Como trabalho futuro, pretende-se em um curto prazo aprimorar o algoritmo de predição que armazena e analisa os valores recebidos pelo sensor de fluxo, assim como, adicionar novas variáveis de análise do paciente como idade, peso e histórico de saúde. Além disso, pretende-se adicionar um sensor de pressão para assegurar maior conformidade em relação aos requisitos do sistema.

\section{Referências}

Anvisa (2020). Nota sobre ventiladores pulmonares. http://portal.anvisa.gov.br. Acesso em: 20/08/2020.

Liu, X., Zhang, T., Hu, N., Zhang, P., and Zhang, Y. (2020). The method of internet of things access and network communication based on mqtt. Computer Communications, 153:169 - 176 .

Naik, N. (2017). Choice of effective messaging protocols for iot systems: Mqtt, coap, amqp and http. In 2017 IEEE International Systems Engineering Symposium (ISSE), pages $1-7$.

OnOff (2020). Iot onoff. https://www.iot-onoff.com (acessado em 10/06/2020).

Schena, E., Massaroni, C., Saccomandi, P., and Cecchini, S. (2015). Flow measurement in mechanical ventilation: A review. Medical Engineering and Physics, 37(3):257-264.

Sharon, Y., Khachatryan, B., and Cheskis, D. (2018). Towards a low current hall effect sensor. Sensors and Actuators A: Physical, 279:278 - 283.

Silva, D. M. and Moraes, R. (2007). Sistema para ensaio de desempenho de ventiladores pulmonares. Master's thesis, Universidade Federal de Santa Catarina, Centro Tecnológico. Programa de Pós-Graduação em Engenharia Elétrica.

Silva, P. L. and Rocco, P. R. M. The basics of respiratory mechanics: ventilator-derived parameters. Annals of Translational Medicine, 6(19).

Uechi, C. A. S., da Silva, J. F., and da Rocha, A. F. (2012). Confiabilidade metrológica de ventiladores pulmonares para cuidados críticos. Master's thesis, Engenharia Biomédica - Universidade de Brasília.

UK-Government (2020). Specification for ventilators to be used in uk hospitals during the coronavirus (covid-19) outbreak. http://bit.ly/UK-RMVS (acessado em 01/07/2020).

Yang, W., Cao, Q., Qin, L., Wang, X., Cheng, Z., Pan, A., Dai, J., Sun, Q., Zhao, F., Qu, J., and Yan, F. (2020). Clinical characteristics and imaging manifestations of the 2019 novel coronavirus disease (covid-19): A multi-center study in wenzhou city, zhejiang, china. Journal of Infection, 80(4):388 - 393. 